\title{
COT-1 BANDING OF HUMAN CHROMOSOMES USING FLUORESCENCE IN SITU HYBRIDIZATION WITH CY3 LABELING
}

\author{
Yimin Wang, Shinsei Minoshima, and Nobuyoshi Shimizu* \\ Department of Molecular Biology, Keio University School of Medicine, \\ 35 Shinanomachi, Shinjuku-ku, Tokyo 160, Japan
}

\begin{abstract}
Summary We developed a new chromosome banding method by in situ hybridization of human Cot-1 DNA as a probe. Clear banding was produced on metaphase chromosomes of lymphoblastoid cells after probe detection with a fluorescent dye Cy3. Comparison with the known banding patterns revealed a similarity to the R-banding with some significant differences: some centromeric heterochromatin regions show Cot-1 positive bands. This suggests that some repetitive sequences from the heterochromatin regions constitute a major component of Cot-1 DNA. This unique chromosome banding method, Cot-1 banding, may be used as a supplement to the conventional karyotype analysis. Scanning analysis of the fluorescence intensities of Cot-1 banding and Q-banding are useful for objectively analyzing the banding pattern including a detection of chromosome aberrations. The Cot- 1 banding with $\mathrm{Cy} 3$ is particularly powerful when applied for the gene mapping by fluorescence in situ hybridization (FISH) because red fluorescence of $\mathrm{Cy} 3$ for chromosome staining can be readily distinguished from green fluorescence of fluorescein isothiocyanate (FITC) for probe labeling. Using this novel method, we mapped a 4 kb-DNA fragment from myelin protein zero (MPZ) gene on the chromosome 1q22 to q23.

Key Words human Cot-1 DNA, FISH, Cy3, chromosome banding, heterochromatin region
\end{abstract}

\section{INTRODUCTION}

Human Cot-1 DNA is a mixture of highly repetitive DNA sequences such as the $A l u \mathrm{I}$ and KpnI family members (Britten et al., 1974; Weiner et al., 1986) and

Received January 23, 1995; Revised version accepted May 23, 1995.

* To whom correspondence should be addressed. 
little is known about its entire components. It is often used for Southern blotting as an effective reagent to enhance specific binding of DNA probes to human genomic DNA immobilized on membrane filters. More recently, Cot-1 DNA is used for in situ hybridization to suppress non-specific binding of genomic DNA probes to chromosome spreads (Lichter et al., 1988, 1990a, 1990b). Particularly, its use for gene mapping by the fluorescence in situ hybridization (FISH) becomes increasingly important. However, little is known about the relationship between the human Cot-1 DNA and the conventional chromosome banding such as R-banding, Q-banding as well as G-banding. Here, we analyzed the distribution of Cot-1 DNA sequences on human metaphase chromosomes and found the similarity to R-banding. Furthermore, a novel FISH gene mapping protocol was developed using the Cot-1 banding with fluorescent dye Cy3 and FITC detection of gene probe.

\section{MATERIALS AND METHODS}

Fluorescence in situ hybridization. Metaphase chromosome preparation was made from human diploid B-lymphoblastoid cells GM130B by the standard method (Gill et al., 1985). The karyotype of GM130B cell line appears normal and FISH was carried out as previously described (Fukuyama et al., 1991; Wang et al., 1993) with several modifications. Briefly, human Cot-1 DNA (GIBCO BRL) and the גHPO1 clone (containing a $4 \mathrm{~kb}$-fragment of $\mathrm{MPZ}$ gene) were labeled with biotin7-dATP (GIBCO BRL) and digoxigenin-11-dUTP, respectively. This was done by nick translation at $16^{\circ} \mathrm{C}$ for $90 \mathrm{~min}$ in a reaction mixture $(25 \mu \mathrm{l})$ containing 1 U DNA polymerase I (BRL, Gaithersburg, Maryland); 100 pg DNase I (Sigma, St. Louis, Missouri); $20 \mu \mathrm{M}$ each of dCTP, dGTP, dTTP, and biotin-7-dATP. Ten ng of probe DNA was mixed with $10 \mu \mathrm{g}$ of salmon sperm DNA in $10 \mu \mathrm{l}$ of hybridization solution and used for one hybridization sample. For MPZ gene mapping by Cot-1 banding, $120 \mathrm{ng}$ of $\lambda \mathrm{HPO} 1$ probe DNA and $50 \mathrm{ng}$ Cot-1 DNA was mixed for one hybridization. Signal detection of hybridization was carried out using Cy3-conjugated avidin, FITC-conjugated sheep anti-digoxigenin antibody and FITC-conjugated donkey anti-sheep immunoglobulin antibody. Cy3 (Biological Detection Systems, Inc.) labeling with avidin was carried out according to the manufacturer's manual. Fluorescence images of probe hybridization were analyzed by confocal laser scanning microscope MRC-600 (Bio-Rad, Richmond, California). Chromosome spreads with good Cot-1 banding were closely examined and their positions on the slide were recorded by the aid of the ruler on the microscope.

$Q$-banding after Cot-1 banding. After recording the Cot-1 banding image, slides were washed with McIlvaine's buffer ( $\mathrm{pH} \mathrm{5.0)}$ for $10 \mathrm{~min}$, then stained with quinacrine mustard (QM, $20 \mu \mathrm{g} / \mathrm{ml}$, Sigma) in Mcllvaine's buffer for $20 \mathrm{~min}$ and washed in the same buffer for another $20 \mathrm{~min}$. Thus, two different fluorescent images, Cy3 fluorescent signals from Cot-1 DNA hybridization and Q-banded 
chromosomes, were obtained from the same chromosome spread. Post-hybridization Q-banding was not performed for the chromosome sample with MPZ gene hybridization.

Merging two fuorescent images. By utilizing the MRC-600 COMOS software, it is feasible to objectively merge two fluorescent images from the same chromosome spread (Wang et al., 1993, 1994). As a result, three colors can be seen on the merged image: red from Cy3-detected Cot-1 DNA sequences; green from Q-banding and yellow from overlapping of two colors.

Analysis of fluorescence intensities from Cot- 1 banding and Q-banding. For detailed analysis of Cot-1 banding and an objective comparison with Q-banding, fluorescence profile of each chromosome was scanned by the COMOS software and analyzed using a software Cricket Graph (CA). The profiles of Cot-1 banding and Q-banding were superimposed on computer graphics and compared.

\section{RESULTS}

\section{Karyotype analysis of Cot-1 banding and other bandings}

Human metaphase chromosomes were in situ hybridized with biotin-labeled human Cot-1 DNA and hybridization was detected by avidin-Cy3 under confocal laser microscope. Distinct banding was revealed on each of 46 human chromosomes (Fig. 1A). There is high contrast between positive and negative bands. This Cot-1 banding image was recorded in a computer, and then Q-banding was performed on the same spread by conventional method (Fig. 1B). Then, these two fluorescent images of Cot-1 banding and Q-banding were computer-graphically merged (Fig. 1C). It is clearly seen that 46 chromosomes display three color images: red reflecting Cot-1 banding, green from Q-banding and yellow indicating overlaps of Cot-1 banding and Q-banding. The distinct red and green bands represent the uniqueness of each banding. The karyotype of a single metaphase spread is shown in these three different ways (Fig. 1D). Also, Cot-1 banding was compared with typical R-banding and $A$ lu-hybridization pattern (Fig. 2). Strong Cot-1 positive bands are found in the centromeric heterochromatin regions of chromosomes $1,3,9,13,14,15,16,19,21,22$, and $Y$, whereas in R-banding the centromeric positive bands can be seen only on the acrocentric chromosomes (13, $14,15,21$, and 22) and not on the other chromosomes (1, 3, 9, 16, 19, and Y).

\section{Scanning analysis of fuorescent intensities from Cot-1 banding and $Q$-banding}

Comparison of the fluorescent intensities of Cot-1 banding and Q-banding on the same chromosome spread by scanning graphs made two banding patterns more distinct (Fig. 3). More than 20 positive bands (peaks of graphs) were observed for chromosome 1. Obvious Q-positive bands were seen on the p35 to $\mathrm{p} 34, \mathrm{p} 33, \mathrm{p} 31, \mathrm{p} 21, \mathrm{q} 22, \mathrm{q} 24, \mathrm{q} 31, \mathrm{q} 41$, and $\mathrm{q} 43$, while Cot-1 positive bands were predominant on $\mathrm{p} 36, \mathrm{p} 35$ to $\mathrm{p} 34, \mathrm{p} 32$ to $\mathrm{p} 22, \mathrm{p} 21$ to $\mathrm{p} 13, \mathrm{q} 1$ to $\mathrm{q} 21, \mathrm{q} 22$ to $\mathrm{q} 24, \mathrm{q} 25$, 


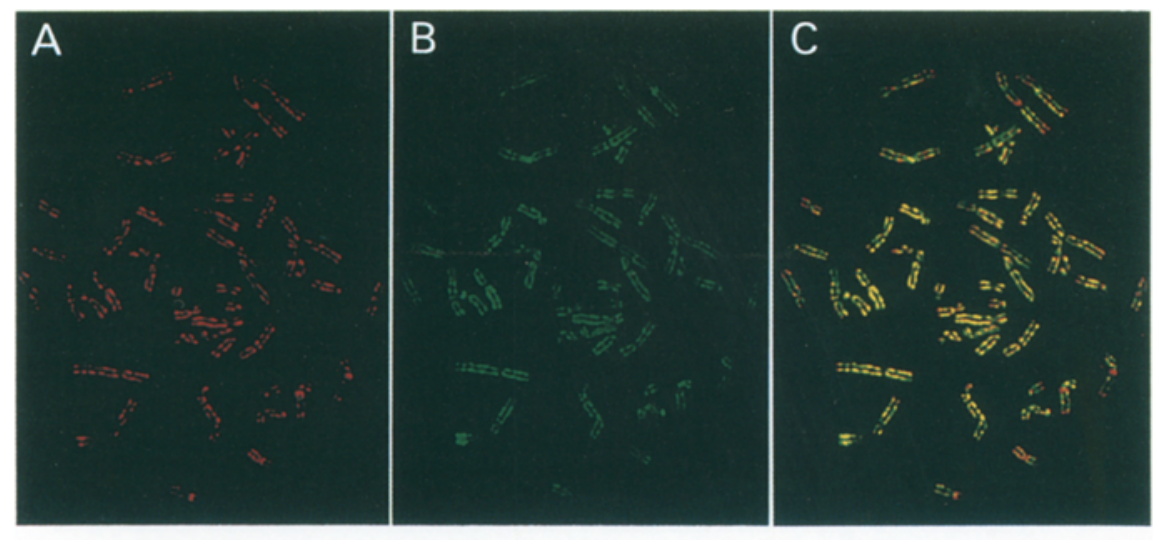

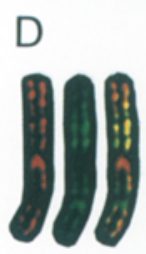

1

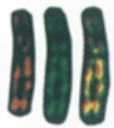

6

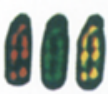

13

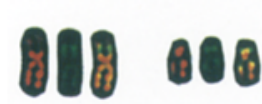

19

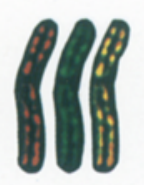

2

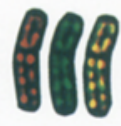

7

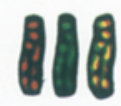

14

20

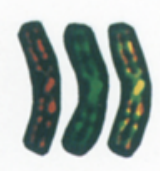

3

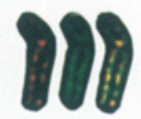

8

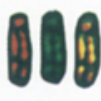

15

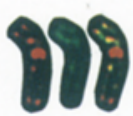

9

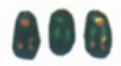

21

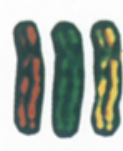

4

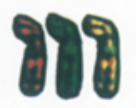

10

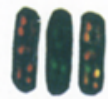

16

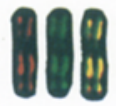

11

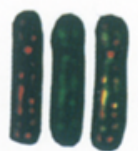

5

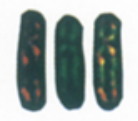

12

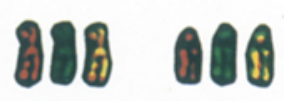

17

18

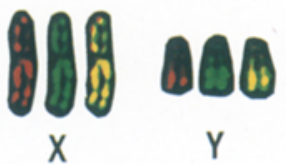

Fig. 1. A: Distribution of Cot-1 DNA sequences on human metaphase chromosomes detected by a fluorescent dye Cy3. Biotin-labeled human Cot-1 DNA probe was hybridized to metaphase chromosomes made from GM130B lymphoblastoid cell line. After incubation with avidin-conjugated Cy3, clear red bands appeared on 46 chromosomes of the metaphase spread. B: Q-banding pattern; The chromosome spread shown in Fig. A was processed for Q-banding. C: The image computer-graphically merged from Figs. A and B. D: Comparison of the karyotypes between Cot-1 banding and Q-banding. Left: Cot-1 DNA banding, middle: Q-banding, right: the merged image. 


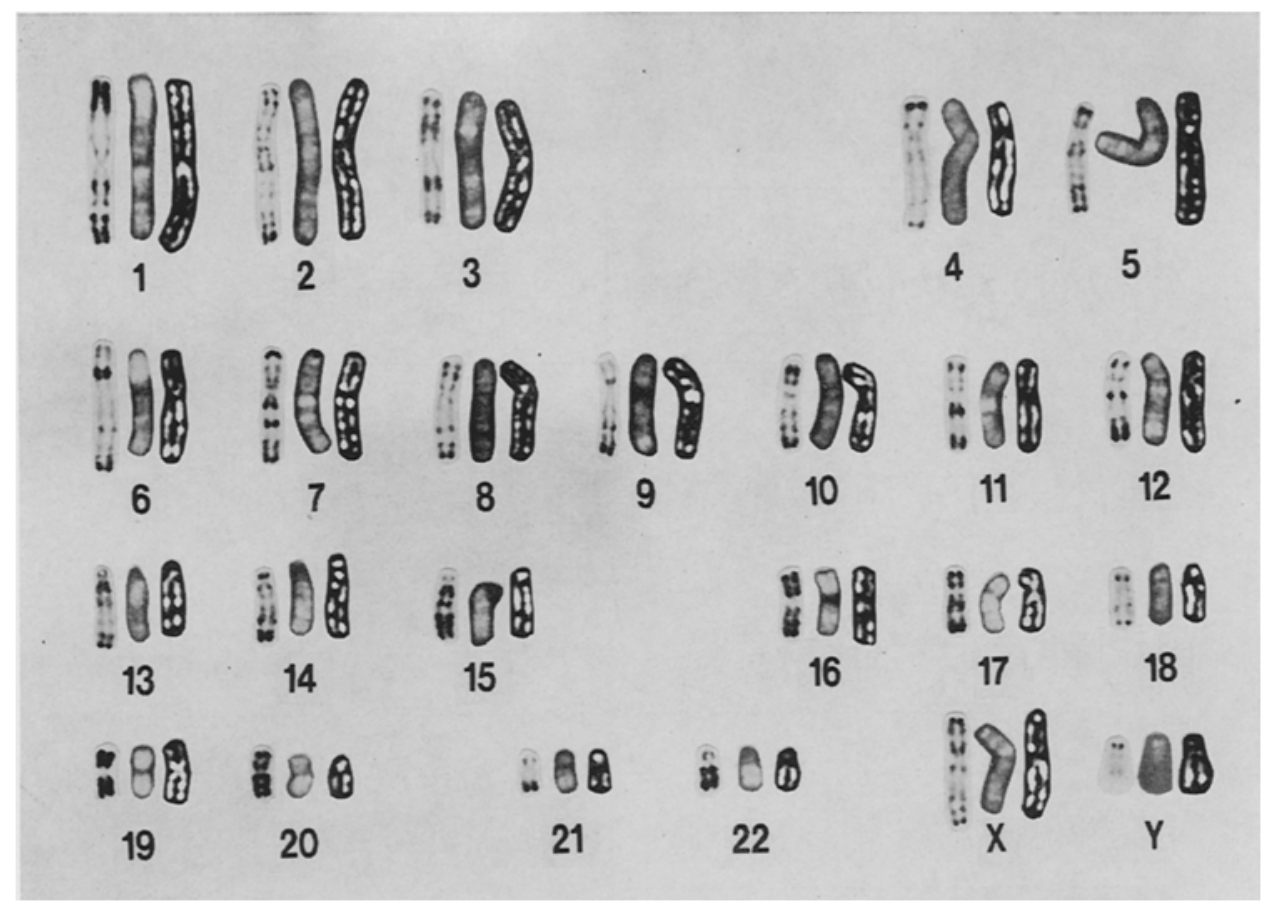

Fig. 2. Comparison of the karyotypes between Cot-1 banding, R-banding and $A l u$-banding. Left: a standard R-banded chromosome (reproduced from Dutrillaux and Prieur, 1978), middle: the $A l u$ hybridization pattern (cited from Korenberg and Rykowski, 1988), right: Cot-1 banding.

$\mathrm{q} 32$, $\mathrm{q} 42$ and $\mathrm{q} 43$ to $\mathrm{q} 44$. All the other chromosomes are with similar banding levels. With the aid of a scanning software, chromosome banding was monitored more quantitatively so that some fine bands were revealed.

\section{Application of Cot-1 banding for gene mapping}

In situ hybridization of metaphase chromosomes were performed using mixed probes of biotin-labeled Cot-1 DNA and digoxigenin-labeled $\lambda$ HPOI DNA (MPZ gene). After hybridization detection, clear FITC signals and Cot-1 banding were observed simultaneously. Two pairs of FITC signals for MPZ gene were seen on the two Cot-1 banded chromosome 1's (Fig. 4). Analyzing the banding pattern with scanning software, MPZ gene was assigned to the band q22 to q23 of chromosome 1. This mapping is consistent with our previous data using G-banding and FISH (Hayasaka et al., 1993; see also Fig. 3). 


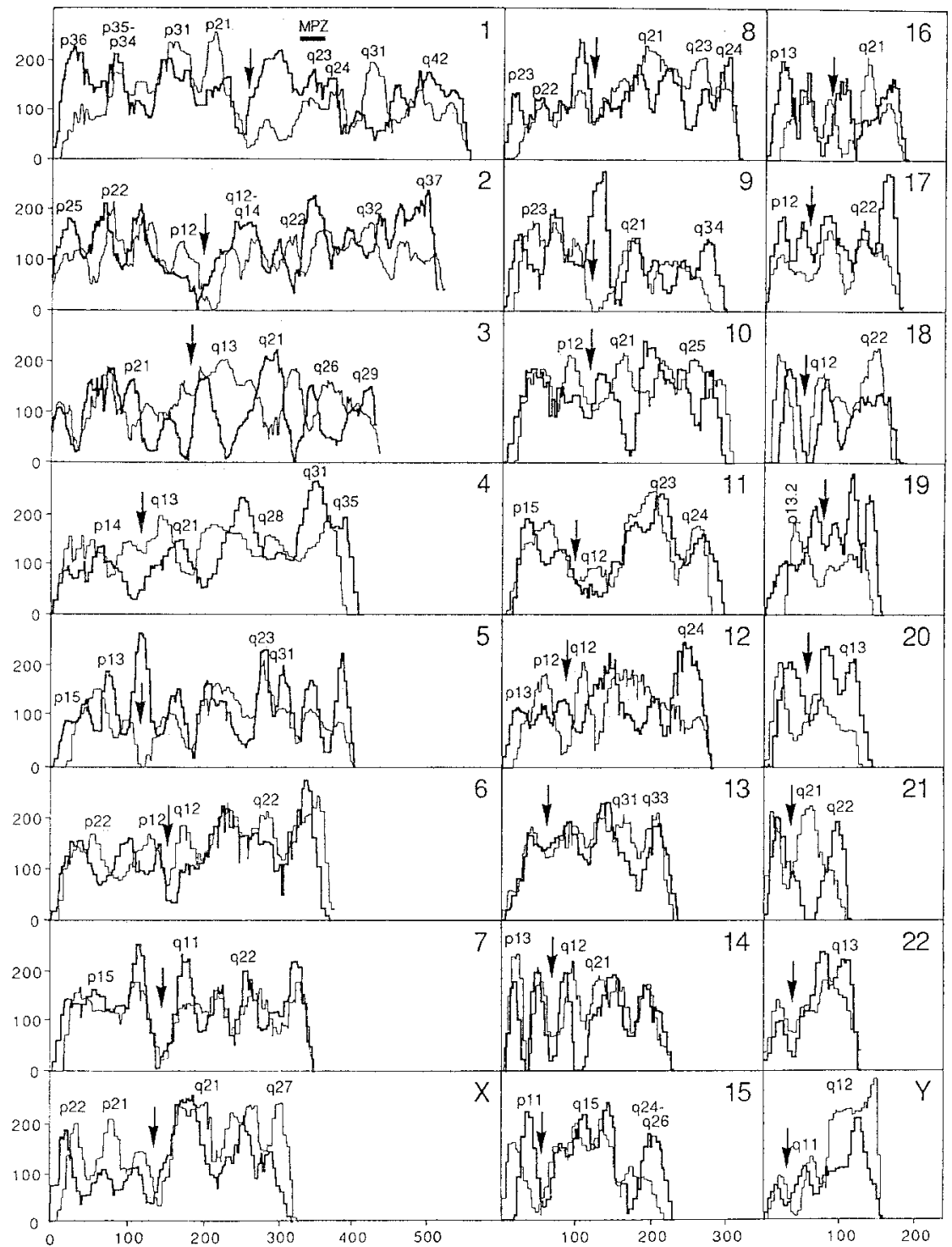

Fig. 3. Scan of fluorescent intensities from 24 different chromatids of metaphase chromosomes. Thick line, Cot-1 banding; thin line, $\mathrm{Q}$-banding. $\mathrm{Y}$ axis and $\mathrm{X}$ axis of each frame represent relative values of fluorescent intensities and chromosome lengths, respectively. Chromosomes are numbered on the right top corner of each frame. Arrows point to the centromere positions. Left side of the arrow is short arm and right side is long arm. Some obvious bands are marked based on Q-banding. 


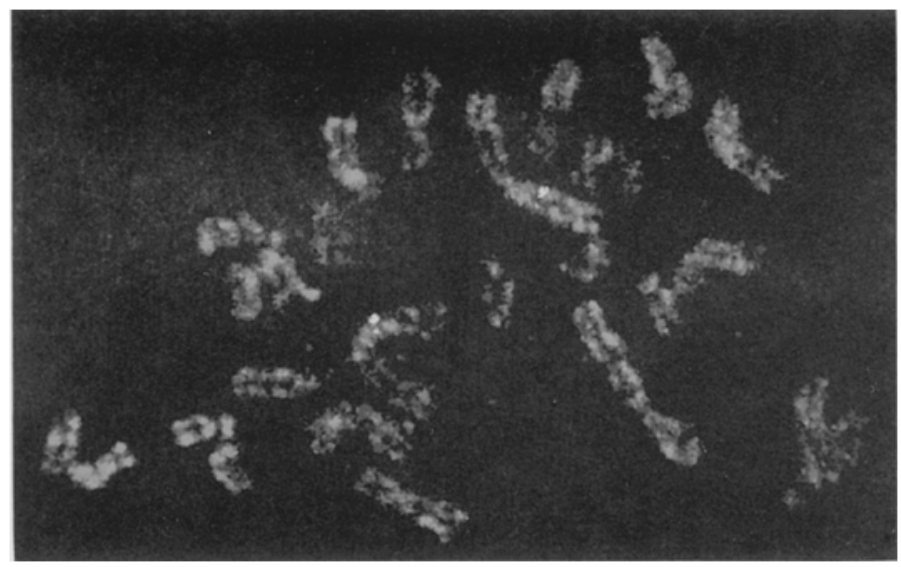

Fig. 4. FISH mapping of MPZ gene on Cot-1 banded chromosomes. Two pairs of FITC signals can be seen on the long arm of chromosome 1 . The hybridization signals were assigned to the q22 to q23 of chromosome 1 by scanning graphs of Cot-1 banding.

\section{DISCUSSION}

Human Cot-1 DNA prepared from placental DNA is known to be highly enriched in repetitive sequences such as the $A l u \mathrm{I}$ and $K p n \mathrm{I}$ family members (Britten et al., 1974; Weiner et al., 1986; Nisson et al., 1991). It is conventionally used to suppress hybridization signals from repetitive DNA sequences present in the genomic DNA probe when Southern blots or in situ hybridization are performed. Here, we used Cot-1 DNA as a hybridization probe for FISH to investigate the distribution of Cot-1 DNA sequences on human metaphase chromosomes and compared the banding pattern with Q-banding, R-banding and Alu hybridization pattern. Our analysis revealed that human Cot-1 DNA hybridization provides a new way to obtain chromosome banding. The Cot-1 banding pattern is similar to R-banding, but with some significant differences. Most obvious difference between Cot-1 banding and R-banding is found in the heterochromatin regions. The Cot-1 banding shows positive bands in the heterochromatin regions known for 11 different chromosomes, whereas R-banding shows positive bands in the heterochromatin regions only on the $\mathrm{D}$ and $\mathrm{G}$ group chromosomes. Alu banding pattern appears similar to R-banding (Baldini and Ward, 1991). No obviously positive $A l u$ bands were found on the heterochromatin regions. It is therefore suggested that repetitive sequences other than $A l u$ or KpnI are apparently contained in the human Cot-1 DNA preparation and caused the positive bands on the heterochromatin regions. The banding pattern obtained from Cot-1 DNA hybridization to metaphase chromosomes is reproducible and may be used as a supplement to the conventional karyotype analysis. 
The scanning analysis of the fluorescence intensity is useful in quantifying a banding pattern and the resolution is sufficient for locating a hybridization signal on banded chromosomes. This could be useful for more precise analysis of chromosome aberrations and gene localization. The scanning analysis revealed that some Cot-1 bands and Q-bands are overlapped as seen for the p35 to p34, p31, and $\mathrm{q} 24$ of chromosome 1 (Fig. 3). One possibility might be the merger of bands or sub-bands in a particular region. For example, big Cot-1 positive band on the q12 to q14 of chromosome 2 might be resulted from the merger of q13 and q14.2 that overlapped with the Q-positive bands of q12 and q14. Many other overlapped bands between Cot-1 and Q-banding are thought to be from the same reasons. Another possibility is that Cot-1 DNA partially hybridizes to Q-positive bands. It has been documented that the SINEs (short interspersed repeated sequences) such as $A l u$ family dominates in the R-bands and the LINEs (long interspersed repeated sequences) such as KpnI (Ll) family dominates in the Q-positive bands (Korenberg and Rykowski, 1988; Holmquist et al., 1988). The L1 family is also contained in Cot- 1 DNA, therefore the partial Cot-1 staining of Q-positive bands seems reasonable. However, we could not see any significant Cot-1 bands on the Q-positive band positions from human Cot-1 DNA hybridization. This is probably due to insufficient contents of $\mathrm{L} 1$ component in the Cot-1 DNA.

Recently, gene mapping by FISH method has become increasingly important in human genome analysis. Banding techniques are essential for fine mapping. The R-banding, Q-banding, G-banding and DAPI staining are often used for FISH mapping (Bhatt et al., 1988; Viegas-Pequignot et al., 1989; Takahashi et al., 1991). Chromosome banding after DNA probe hybridization is an objective way to localize a gene at cytogenetic resolution levels. Q-banding has been successfully used for this purpose by performing probe detection with Texas red (Fukuyama and Shimizu, 1992). FITC is commonly used to detect probe hybridization and is considered to be a stable fluorescent dye. However, Q-banding and FITC detection cannot be used simultaneously because both FITC and quinacrine generate green fluorescence. Contrary to this, red fluorescence of $\mathrm{Cy} 3$ for staining chromosomes can be readily distinguished from green fluorescence of FITC for probe labeling. Clear green signals of probe DNA on the red banded chromosomes can be obtained and the banding resolution is as clear as Q-banding. By this method, we mapped a 4-kb genomic DNA fragment from myelin protein zero (MPZ) gene on chromosome lq22 to q23 (Fig. 4) that is consistent with our previous data (Hayasaka et al., 1993). The simultaneous detection of biotin-labeled Cot-1 DNA does not affect the sensitivity to detect digoxigenin-labeled marker DNA probe although the probe contains only a 4-kb MPZ gene fragment. Furthermore, fluorescent dye $\mathrm{Cy} 3$ seems much more efficient than FITC when used for detecting the Cot-1 hybridization (unpublished data). The PrI counter-staining of Cot-1 banded chromosomes is also feasible, making Cot-1 negative bands visible and chromosome shape more distinctive (unpublished data). 
The DNA probe localization with Cot-1 banding could be a convenient way of mapping a gene or DNA fragment. Cot-1 banding can serve as a new banding method with some similarity to the R-banding.

Acknowledgments We thank Mr. M. Maekawa and Ms. M. Takata for their technical assistance and also thank Ms. H. Harigai for her excellent assistance in manuscript preparation. This work was supported by a Grant-in-Aid for a Creative Basic Research (Human Genome Program) from the Ministry of Education, Science and Culture of Japan, and the Special Coordination Funds for Promoting Science and Technology from the Science and Technology Agency of Japan.

\section{REFERENCES}

Baldini A, Ward DC (1991): In situ hybridization banding of human chromosomes with Alu-PCR products: a simultaneous karyotype for gene mapping studies. Genomics 9: 770-774

Bhatt B, Burns J, Flannery D, McGee J (1988): Direct visualization of single copy genes on banded metaphase chromosomes by nonisotopic in situ hybridization. Nucleic Acids Res 16: 39513961

Britten RJ, Graham DE, Neufeld BR (1974): Analysis of repeating DNA sequences by reassociation. Methods Enzymol 29: 363-417

Dutrillaux B, Prieur M (1978): The normal human karyotype G and R bands. In: ISCN (1978): An International System for Human Cytogenetic Nomenclature (1978). Birth Defects: Original Article Series, Vol. XIV, No 8 (The National Foundation, New York 1978); also in Cytogenet Cell Genet 21: $309-404$ (1978)

Fukuyama R, Ichijou Y, Minoshima S, Kitamura N, Shimizu N (1991): Regional localization of the hepatocyte growth factor (HGF) gene to human chromosome 7 band q21.1. Genomics 11: $410-415$

Fukuyama R, Shimizu N (1992): Detection of variation in the ribosomal RNA gene clusters by a modified fluorescence in situ hybridization method. Jpn J Human Genet 37: 139-143

Gill GN, Weber W, Thompson DM, Lin C, Evans RM, Rosenfeld MG, Gamou S, Shimizu N (1985): Relationship between production of epidermal growth factor receptors, gene amplification, and chromosome 7 translocation in variant A431 cell. Somat Cell Mol Genet 11: 309-318

Hayasaka K, Himuro M, Wang Y, Takata M, Minoshima S, Shimizu N, Miura M, Uyemura K, Takada G (1993): Structure and chromosomal localization of the gene encoding the human myelin protein zero (MPZ). Genomics 17: 755-758

Holmquist GP (1988): DNA sequences in G-bands and R-bands. In: Adolph KW (ed). Chromosomes and chromatin. CRC Press, Boca Raton, pp 76-121

Korenberg JR, Rykowski MC (1988): Human genome organization: Alu, Lines, and the molecular structure of metaphase chromosome bands. Cell 53: 391-400

Lichter P, Cremer T, Borden J, Manuelidis L, Ward DC (1988): Delineation of individual human chromosomes in metaphase and interphase cells by in situ suppression hybridization using recombinant DNA libraries. Hum Genet 80: 224-234

Lichter P, Tang CC, Call K, Hermanson G, Evans GA, Housman D, Ward DC (1990a): Highresolution mapping of human chromosome 11 by in situ hybridization with cosmid clones. Science 247: 64-69

Lichter P, Ledbetter SA, Ledbetter DH, Ward DC (1990b): Fluorescence in situ hybridization with Alu and L1 polymerase chain reaction probes for rapid characterization of human chromosomes in hybrid cell lines. Proc Natl Acad Sci USA 87: 6634-6638

Nisson PE, Watkins PC, Menninger JC, Ward DC (1991): Improved suppression hybridization with human DNA (Cot-1 DNA) enriched for repetitive DNA sequences. Focus 13: 42-45 
Takahashi E, Hori T, O'Connell P, Leppert M, White R (1991): Mapping of the MYC gene to band 8q24.12-q24.13 by R-banding and distal to fra(8)(q24.11), FRA8E, by fluorescence in situ hybridization. Cytogenet Cell Genet 57: 109-111

Viegas-Pequignot E, Dutrillaux B, Magdelenat H, Coppey-Moisan M (1989): Mapping of singlecopy DNA sequences on human chromosomes by in situ hybridization with biotinylated probes: enhancement of detection sensitivity by intensified-fluorescence digital-imaging microscopy. Proc Natl Acad Sci USA 86: $582-586$

Wang Y, Minoshima S, Shimizu N (1993): Precise mapping of the EGF receptor gene on the human chromosome 7p12 using an improved FISH technique. Jpn J Human Genet 38: 401-408

Wang Y, Amagai M, Minoshima S, Sakai K, Green KJ, Nishikawa T, Shimizu N (1994): The human genes for desmogleins (DSG1 and DSG3) are located in a small region on chromosome $18 \mathrm{q} 12$. Genomics 20: 492-495

Weiner AM, Deininger PL, Efstratiadis A (1986): Nonviral retroposons: genes, pseudogenes, and transposable elements generated by the reverse flow of genetic information. Annu Rev Biochem 55: 631-661 